\title{
A Novel Acoustic Emission Source Location Method in the Crane Based on EEMD-FastICA
}

\author{
Yu Jiang ${ }^{1 *}$ and Feiyun $\mathrm{Xu}^{2}$ \\ 1. College of Intelligent Science and control Engineering, Jinling Institute of Technology, Nanjing 211169, China \\ 2. School of Mechanical Engineering, Southeast University, Nanjing 211189, China
}

\begin{abstract}
The study presents a novel source location method based on EEMD (ensemble empirical mode decomposition) and optimized FastICA (independent component analysis) for determining the position of the AE (acoustic emission) sources in the damage structure of crane. Firstly, The AE signals are self-adaptive decomposed into a number of IMFs (intrinsic mode functions) by using EEMD algorithm. Then, the main feature IMFs signals are extracted as the effective AE source signal by optimized Fast-ICA method, the PSD (power spectral density) of each IMF and the real IMFs are obtained. According to the principle of spectrum similarity, time delay is computed at the different channels in combination with the Cross Correlation time delay estimation principle. Thirdly, a novel source location algorithm based on EEMD-FastICA is proposed and the results of AE source location are obtained. Finally, the three-point bending test for the crane is implemented in order to validate the efficiency of the proposed method. The experimental results indicate that the proposed method has the ability to determinate the position of the AE sources and reduce the interference noises. Moreover, compared with the traditional location algorithm, a considerable improvement is obtained.
\end{abstract}

Key words: Source location, EEMD, FastICA, cranet, three-point bending test.

\section{Introduction}

AE source location technology can determine the damaged position of the defect,especially of early damage of materials for dynamic monitoring [1, 2]. However, Due to the influence of AE source features, AE signal propagation paths, environmental noises and measurement system, the AE signal is very complex, which is a typical nonlinear and non-stationary random signal and needs to use needle of the signal processing technique to extract the effective information from the AE signal. The EMD (empirical mode decomposition) introduced by Huang [3] is a good time-frequency tool. However, EMD decomposes $\mathrm{AE}$ signal into a number of basic constituent signals called IMFs (intrinsic mode functions), which obtained IMFs is not a strict single component signal, but accompanied by the IMF mode

Corresponding author: Yu Jiang, teacher, Dr., research fields: acoustic emission structural damage detection, signal processing. aliasing and false signals mixed, seriously impacting on the acoustic emission data analysis and acoustic emission source location accuracy. In 2009, Wu and Huang [4] proposed an improved version of EMD, by adding finite white noise to the investigated signal, this improved method called EEMD can eliminate the mode mixing problem of EMD automatically. Based on these merits, the EEMD has lately attracted significant attention and it has been proven to outperform EMD in decomposing random signals. Apart from using EEMD as a non-stationary signal decomposing tool, Guo and Tse $[5,6]$ studied the EEMD, which eases the problem of mode mixing in real AE signals generated from defective area. They performed a series of investigations to reveal the relationship between the amplitude of the added white noise and the number of ensemble members for the minimization of the mode mixing problem, it concluded that a higher number of ensemble members leads to smaller RMS error. Zvokelj [7] et al. proposed the EEMD-MSPCA (EEMD-based multiscale principal 
component analysis) techniques to overcome the non-adaptive nature in conventional MSPCA. They applied their in vibration as well as acoustic emission recordings from large-size slow-speed bearings, it not only highlighted the improvement of signal-to-noise-ratio, but also the enhanced diagnostic capability. The only potential drawback is that the proposed techniques are computationally intensive. However, as a matter of fact, among the approaches reported in the aforementioned literature relies on information coming both from the normal as well as the faulty class. In most real life applications, data from the possible faulty modes are not readily available, making the binary (or multi-class) classification approach very difficult and impractical. The main innovation of this paper stems from the proposal of a combined and integrated anomaly detection approach to signal processing. A novel CC (cross-correlation) signal processing method based on EEMD (ensemble empirical mode decomposition) and Fast-ICA (optimized independent component analysis) is presented for auxiliary determining the position of the AE sources.

\section{Cross-Correlation Source location}

\subsection{Cross-Correlation Algorithm}

Time Delay Estimation is a typical passive method for target parameter estimation. $\mathrm{CC}$ is to use similar signal in time domain for time delay estimation [8], which is the most basic method of time delay estimation. Eq. (1) is AE signal model.

$$
s(t)=A(t) e^{j \theta(t)}
$$

where, $A(t)$ is the envelope amplitude of AE signal, $\theta(t)$ is the frequency and phase of function with the attenuation and oscillation signal. Generally, this model belongs to random distribution form, which reflects related features including the acoustic emission source, propagation medium, and acoustic emission instrument response.

Considering the signal received from the sensor will be affected by the noise interference, the received signal model of the $i^{\text {th }}$ sensor can be assumed as the following $[14,15]$.

$$
x_{i}(n)=\delta_{i} s\left(n-\tau_{i}\right)+v_{i}(n)
$$

where, $\delta_{i}$ is the attenuation coefficient of the acoustic wave propagation $\delta_{i} \in[0,1], \tau_{i}$ is the delay time of acoustic source arrived the $i^{\text {th }}$ sensor and $v_{i}(n)$ is the added noise. According to Eq. (2), Eq. (3) is as following:

$$
\tau_{i j}=\tau_{i}-\tau_{j}
$$

the received signal model of the $j^{\text {th }}$ sensor can be shown as Eq. (4).

$$
\begin{gathered}
x_{j}(n)=\delta_{j} . s\left(n-\tau_{j}\right)+v_{j}(n) \\
=\delta_{j} . s\left(n-\tau_{i}+\tau_{j}\right)+v_{j}(n) \\
=\delta_{i} . s\left(n-\tau_{i j}\right)+v_{j}(n)
\end{gathered}
$$

If $s(n), v_{i}(n)$, and $v_{j}(n)$ are independent of each other, then the cross-correlation function of signal received from the two sensors will be $R_{i j}(\tau)$, namely,

$$
\begin{gathered}
R_{i j}(\tau)=E\left[x_{i}(n) x_{j}(n+\tau)\right] \\
=\delta_{i} \cdot \delta_{j} E\left[s(n) s\left(n+\tau-\tau_{i j}\right)\right] \\
=\delta_{i} \delta_{j} R_{s s}\left(\tau-\tau_{i j}\right)
\end{gathered}
$$

where, $\tau_{i j}$ is the delay time and $R_{s s}$ is the self-correlation function of source signal $s(n)$, based on the self-correlation theory, when $R_{s s}\left(\tau-\tau_{i j}\right) \leq R_{s s}(0)$, the two received signals will have the max value. The peak point of the correction function will be the value of time delay estimation. On this basis, according to the theory of linear location, the time difference of the two sensors from the same acoustic source will be $\tau_{i j}$

$$
\tau_{i j}=\left(L_{i}-L_{j}\right) / c
$$

where, $L_{i}, L_{j}$ is the distance between the acoustic and the two sensors, $c$ is acoustic velocity and $D$ is the 
position of two sensors. Then,

$$
\begin{gathered}
L_{i}=\left(D-c \times \tau_{i j}\right) / 2 \\
L_{j}=D-L_{i}
\end{gathered}
$$

$L_{i}$ is the distance from acoustic source to sensor $\mathrm{i}$.

\subsection{EEMD}

The EMD, originally proposed by Huang [3] in 1998 is a non-linear multi-resolution self-adaptive decomposition technique, which can self-adaptive decompose a complicated signal into a set of complete and almost orthogonal components, IMFs (intrinsic mode functions), without a preliminary knowledge of the nature and the number of IMF components embedded in the data. However, EMD still suffers from the mode mixing problem. To alleviate the drawback of the mode mixing [9], Wu and Huang proposed [3, 4] an effective noise-assisted method named EEMD which significantly reduces the chance of undue mode mixing and preserves the dyadic property of the decomposition for any data. EEMD is an improved version of the original EMD. The principle of the EEMD is simple: The added white noise populates the whole time-frequency space uniformly, facilitating a natural separation of the frequency scales, which reduces the occurrence of mode mixing.

\subsection{Blind Source Separation and Fast-ICA Algorithm}

Blind source separation aims at the false IMFs component separation resulting from the EEMD decomposition, which is a new method of signal processing [10]. The core of this algorithm is to estimate the separation matrix $\mathrm{W}$, and use $y$ as the estimation of the source signal $x(t), y$ shows the output of matrix $W$, namely, as shown in Fig. 1.

$$
s(t)=W x(t)=W A s(t)
$$

The FastICA algorithm is an efficient and popular procedure for independence analysis. The algorithm is

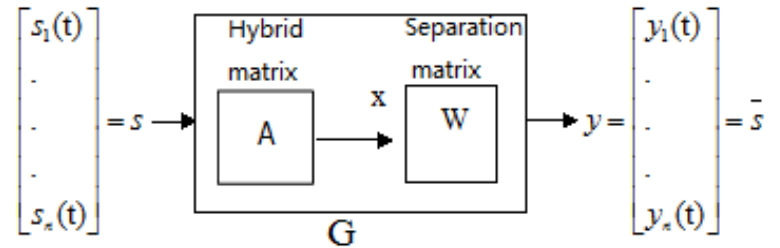

Fig. 1 Blind source separation chart.

maximizing non-Gaussianity using a fixed-point iteration [11-13]. It can also calculate with Newton iteration method. As the first preprocess of the FastICA algorithm, the input vector $\mathrm{X}$ should be centered:

$$
\mathrm{X}=\mathrm{X}-\mathrm{E}\{\mathrm{X}\}
$$

Then, in the second preprocess, the input vector data $\mathrm{X}$ is whitened so that the new components can be uncorrelated and have variance one. Let $\mathrm{c}=\mathrm{E}\left\{\mathrm{XX}^{\mathrm{T}}\right\}$ be the covariance matrix and $\mathrm{d}$ the diagonal matrix of eigenvalues of $\mathrm{c}$. Therefore, the whitened data are:

$$
\tau=\frac{E^{T} X}{\sqrt{d}}
$$

where, $E$ is the matrix of eigenvalues.

The FastICA algorithm finds a projection direction of Eq. (15) or maximizes the non-Gaussianity of the projection $\mathrm{W}^{\mathrm{T}} \tau$ for data $\mathrm{X}$. In this study, due to the extraction of the independent components, the function $f(v)$ is utilized as a non-quadric function with the equation below:

$$
f(v)=\log \cosh (v)
$$

the derivatives $g(v)$ and second derivatives $g^{\prime}(v)$ are:

$$
\begin{gathered}
g(v)=\tanh (v) \\
g^{\prime}(v)=1-\tanh ^{2}(v)
\end{gathered}
$$

By iteratively renewing the unmixing matrix $\mathrm{W}$, the final independent components can be calculated as:

$$
\begin{gathered}
W=E\left\{\tau g\left(W^{T} \tau\right)\right\}-E\left\{g^{\prime}\left(W^{T} \tau\right)\right\} W \\
W=W /\|W\|
\end{gathered}
$$

After every fixed-point iteration, the normalization of $\mathrm{W}$ has been added to improve the stability. When the old and new values of the $\mathrm{W}$ points are in the same 
direction, the fixed-point iteration is converged.

\section{EEMD-Fastica Cross Correlation Source Location Algorithm}

The EEMD-FastICA-CC Source location is an innovation combination algorithm, this method contains three steps: firstly, the signal is decomposed by using the EEMD algorithm; secondly, aiming at the false signal resulting from the EEMD, the Fast ICA algorithm is introduced to realize the decomposition completely and remove the false component decomposition. The higher frequency IMF component that reflects the physical characters of signal is extracted; finally, the combination source location theory and the $\mathrm{CC}$ technique, time delay of the IMF components at different channels is obtained and the positions of AE source are determined. The detailed flowchart is shown in Fig. 2.

\section{Experiment Description}

\subsection{Experiment Layout}

In order to demonstrate the validity of the proposed source location algorithm based on EEMD-FastICA, three point bending experiment is carried out. SJ9-3 of a Q235B (in China Code) mild steel sheet was selected as the experiment sample according to the engineering projects of crane. The specimen area was a $500 \mathrm{~mm} \times$ $60 \mathrm{~mm}$ and the thickness was $8 \mathrm{~mm}$. A weld was in the middle of the sample, two sensors resonant at $150 \mathrm{kHz}$ (DP15I) coupled directly on the face of the sample using vaseline as acoustic couplant (Figs. 3 and 4). Sensor sensitivity was evaluated using the classical PLB (pencil lead break) technique i.e. by means Hsu-Nielsen artificial sources. Germany Zwick/Roell Z100MST, loading velocity was $6 \mathrm{~mm} / \mathrm{min}$. AE activity was recorded using a 48-channel SAMOS PAC equipment. Thresh value is $40 \mathrm{~dB}$, sample rate is 4,096 .

\subsection{Result Analysis}

Seen from Fig. 5a, both energy and impact parameters have the same trend in the two different channels.

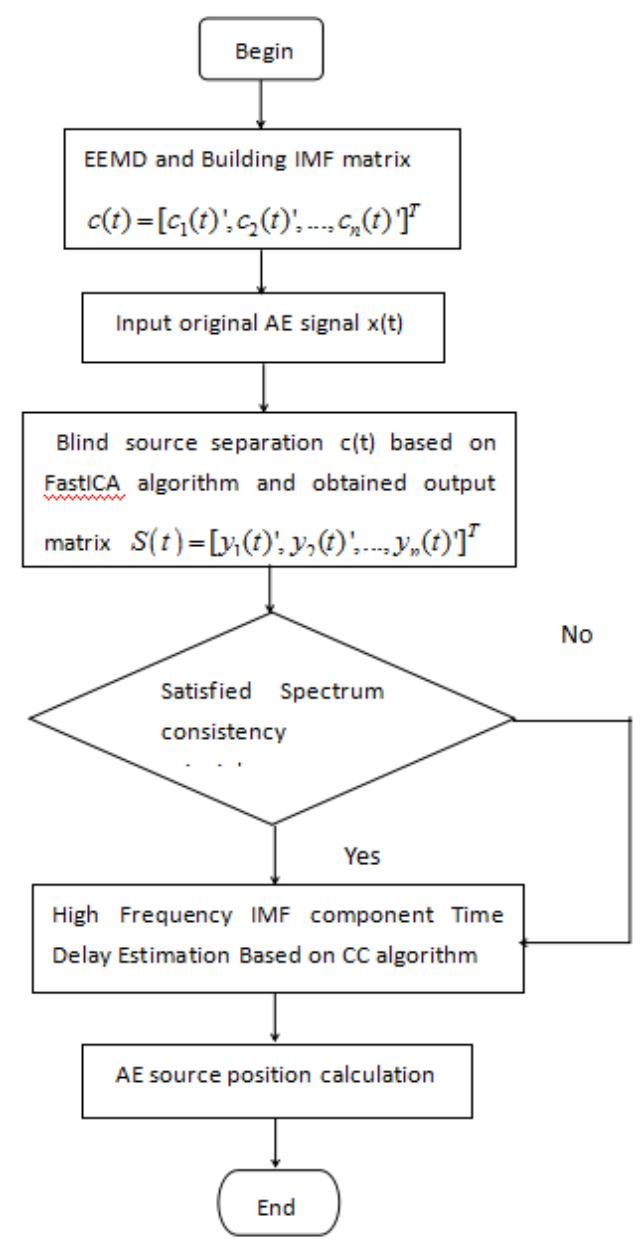

Fig. 2 EEMD-FastICA-CC algorithm flow chart.

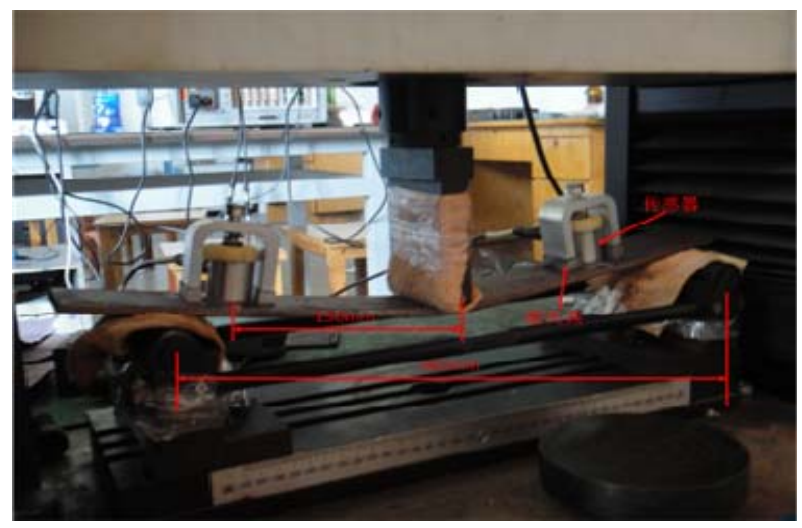

Fig. 3 Three point bending test.

So taking the first channel for example, AE dynamic performance is described by the data analysis. Seen from Fig. 5b, the experiment has four different stages from the loading curve during the whole loading course: elastic stage, plasticity and yield stage, crack propagation stage and instability stage. 


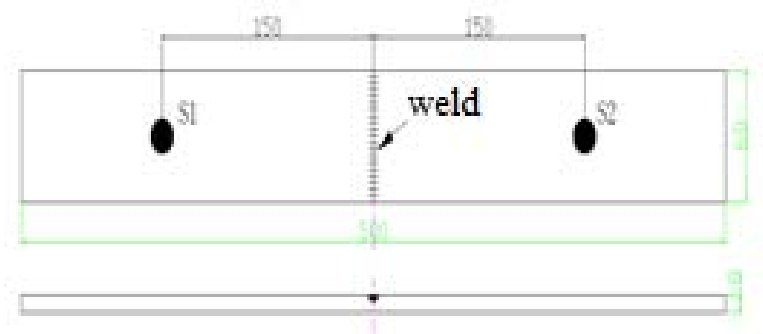

Fig. 4 Q235B welding sample.

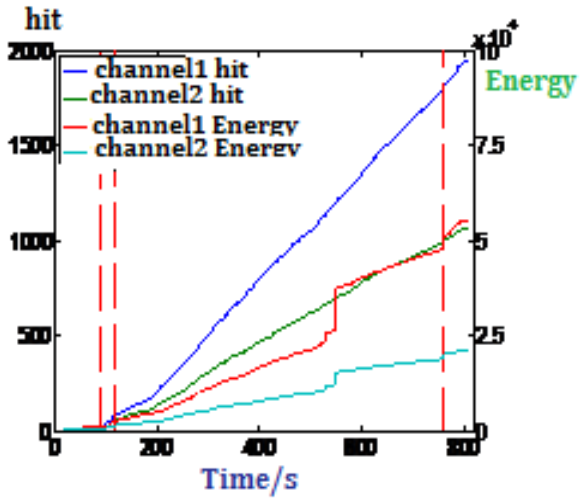

(a) hit vs time

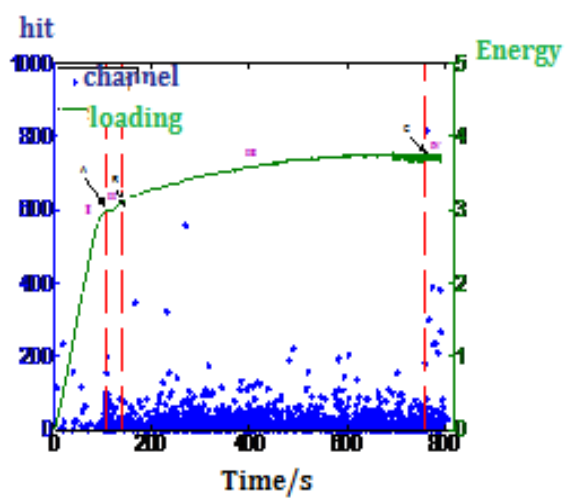

(b) Energy vs time

Fig. 5 AE Performance parameters for (a) and (b).

The first is the elastic stage (I), and this course has a little AE signal and sparsely the energy distribution, acoustic source is mainly noise source.

In the Plasticity and yield stage (II), AE signal has the trend of stable growth and the curvature varies obviously from point $\mathrm{A}$ to point $\mathrm{B}$. This result shows that the position of welding at the area of the stress concentrated begins to produce the plastic deformation and quickly into the yield stage, the energy distribution is still sparse, even the amplitude of energy has an downwards tendency, point $\mathrm{A}$ and point $\mathrm{B}$ corresponding to the upper and lower yield points respectively. Acoustic source is mainly continuous AE source.

In the crack propagation stage (III), the distribution of energy is both gradually intensive and steady growth and the macro-crack occurs initial deformation. At this moment, although the speed of the loading is on the decline, the crack is still rapidly and sharply expanding. After the stress of this peak, the loading velocity keeps this constant value to make the sample reach a maximum bending size that Universal Test Machine allowed and stop loading. At this moment, the crack is still growing. Acoustic source is mostly sudden AE source.

In the instability stage (IV), the crack is in the unstable state till the test ends. Acoustic source is interrupted continuous AE source, complex and unstable. According to the above four different stages of $\mathrm{AE}$ source character analysis, taking the crack propagation stage for example, two channel (S1, S2) AE signals are extracted for the proposed new algorithm. Fig. 6 is the crack signals of S1 and S2.

Seen from Fig. 7, eleven different frequency IMF components and a residual item are obtained by using the EEMD, according to the IMF performance indexes above mentioned literature [10], the reasonable threshold value is 0.02 . The first three IMF components of S1 and $\mathrm{S} 2$ in the crack propagation stage are extracted to reset input maxis for FastICA algorithm, respectively. The results of algorithm are shown as in Figs. 8 and 9, which show the signals of the first three IMF and 


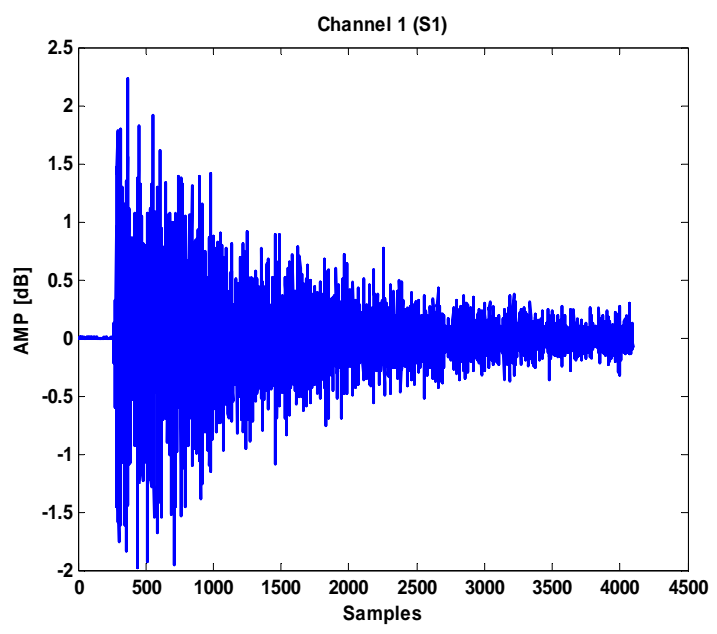

(a) crack signal S1

Fig. 6 Crack propagation stage AE signals for (a) and (b).

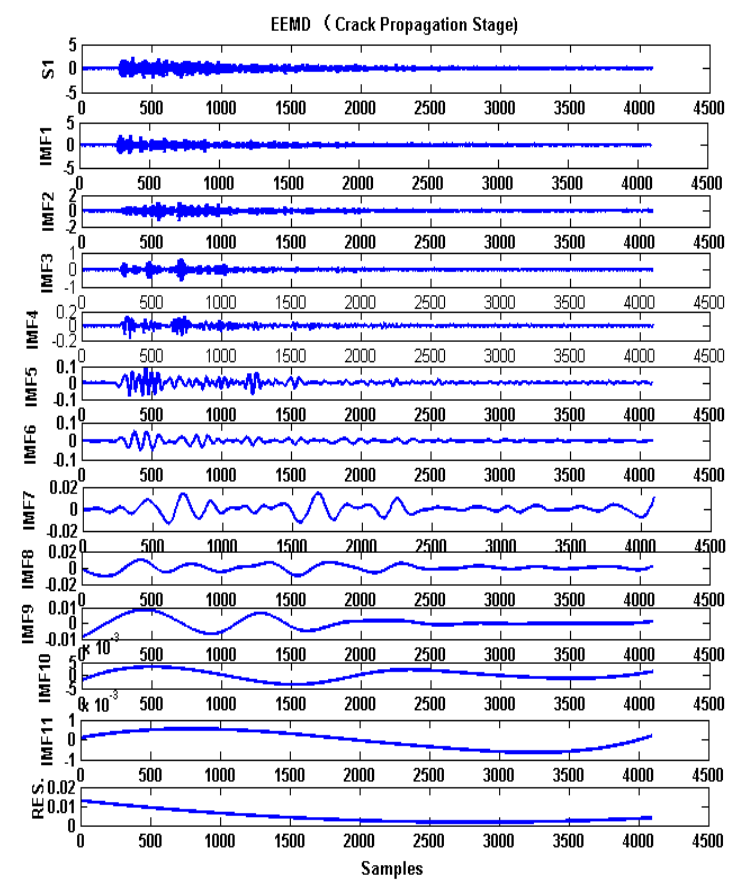

S1-EEMD

Fig. 7 AE signals EEMD for (a) and (b).

FastICA IMF. Figs. 10 and 11 are the corresponding Power Spectrum Distribution.

In order to realize $\mathrm{AE}$ source location, high frequency IMF components of S1 and S2 in FastICA algorithm are extracted with Energy-ratio and spectral similarity factors [14]. Seen from Tables 1 and 2, the signals of Fast-ICA-IMF3 and Fast-ICA-IMF22 are used to perform the cross-correlation algorithm (Fig.

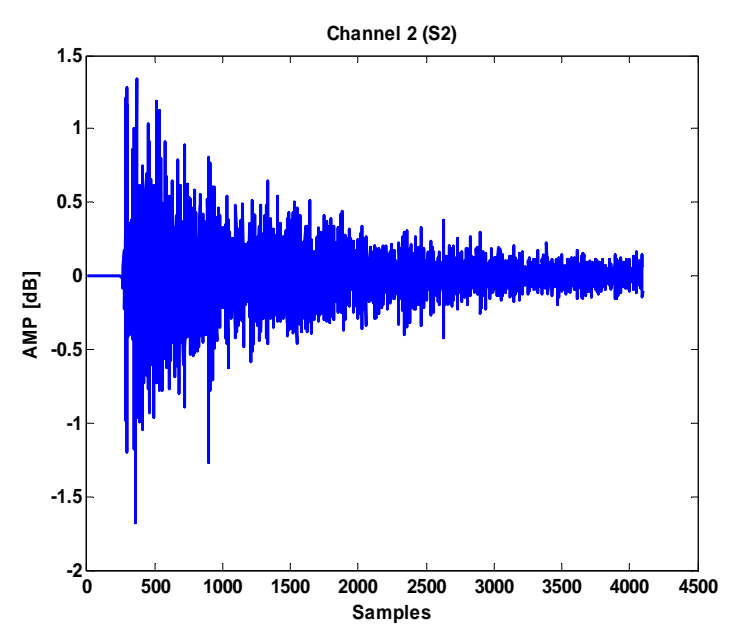

(b) crack signal S2

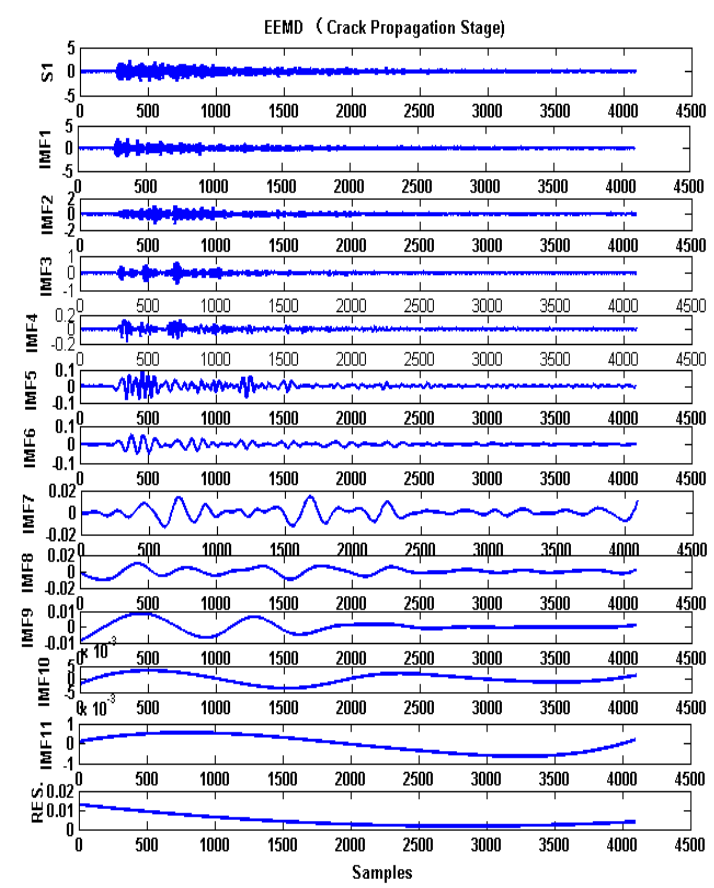

S2-EEMD

10). Finally, according to time delay estimation of cross-correlation $\tau$ and velocity measured $v$ at the beginning of experiment, $\mathrm{AE}$ source location far from sensor S1 is $149.45 \mathrm{~mm}$ in the crack propagation stage by using the linear location calculation, compared to the actual AE loading point location $(150 \mathrm{~mm})$, the error of the location is $0.36 \%(0.546 \mathrm{~mm})$. It shows that the proposed algorithm is very effective and feasible. 


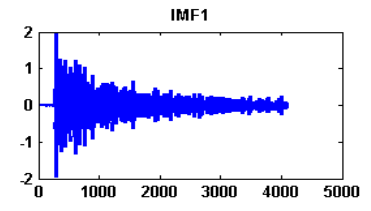

IMF2

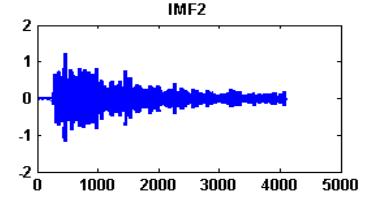

IMF3

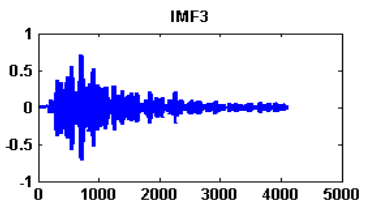

(a) FastICA IMF1-IMF3 (S1)

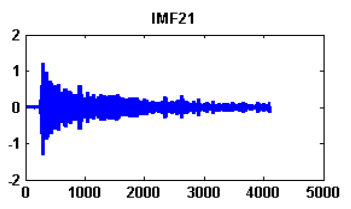

IMF22

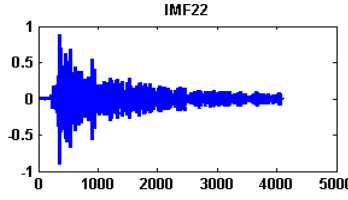

IMF23

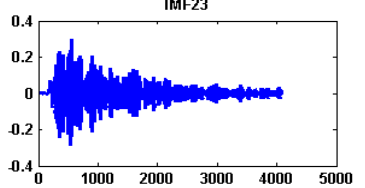

(b) FastICA IMF21-IMF23 (S2)

Fig. 8 FastICA signals processing.
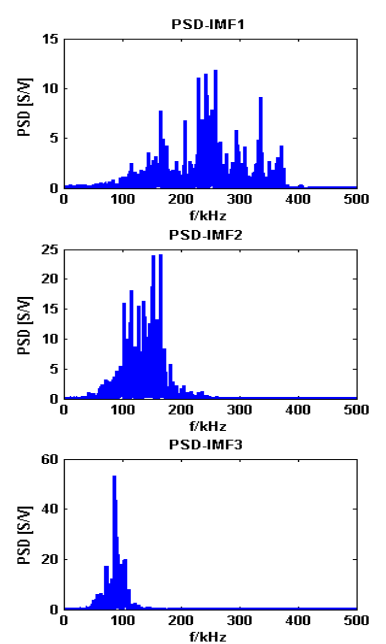

(a) PSD IMF1-IMF3 (S1)
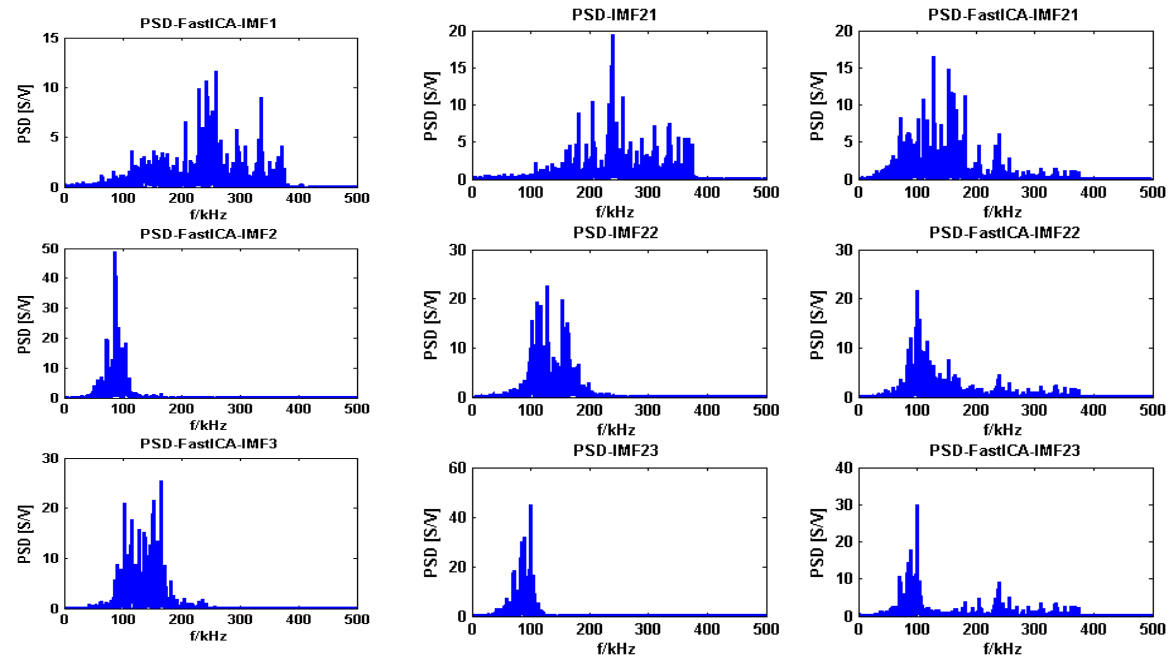

(b) PSD IMF21-IMF23 (S2)
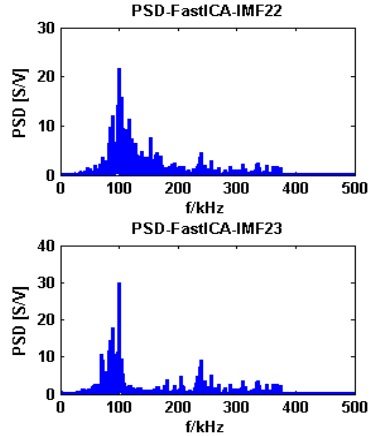

Table 1 High frequency IMF components extracted (S1).

\begin{tabular}{lll}
\hline IMF & Energy-ratio & Spectral similarity \\
\hline FastICA-IMF1 & 2.8844 & 0.6114 \\
FastICA-IMF2 & 2.8845 & 0.7895 \\
FastICA-IMF3 & 2.8871 & 0.9972 \\
\hline
\end{tabular}

Table 2 High frequency IMF components extracted (S2).

\begin{tabular}{lll}
\hline IMF & Energy-ratio & Spectral similarity \\
\hline FastICA-IMF21 & 5.0233 & 0.6150 \\
FastICA-IMF22 & 5.0236 & 0.8606 \\
Fast-ICA-IMF23 & 5.0234 & 0.8359 \\
\hline
\end{tabular}

Fig. 10 Time delay of the EEMD-FastICA CC algorithm. 


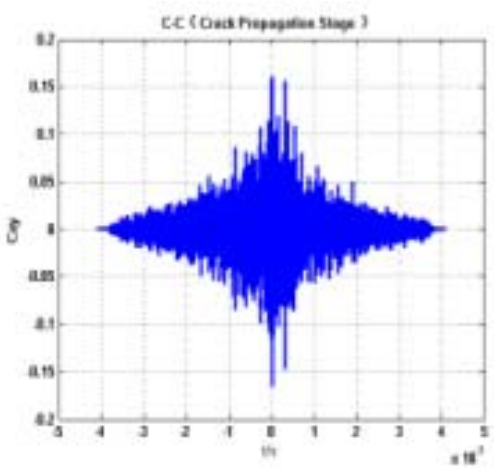

(a) $\mathrm{CC}(\tau=1.8 e-005)$

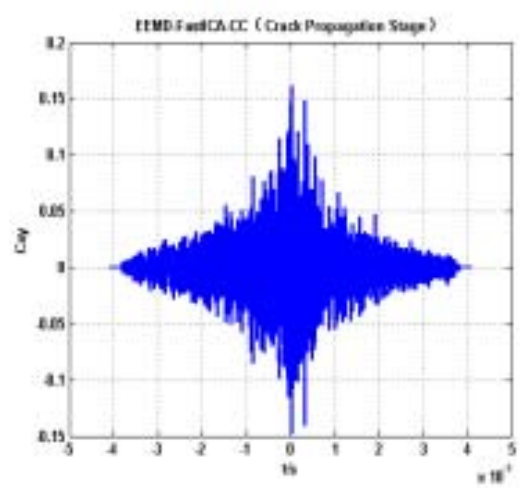

(c) $\operatorname{EEMP}-\mathrm{CC}(\tau=10-005)$

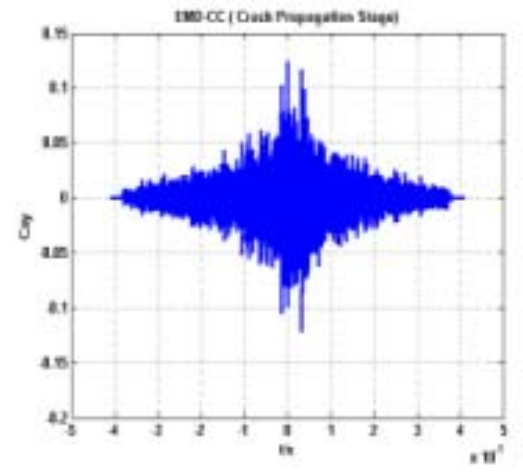

(b) $\operatorname{EMD}-\mathrm{CC}(\tau=1 e-005)$

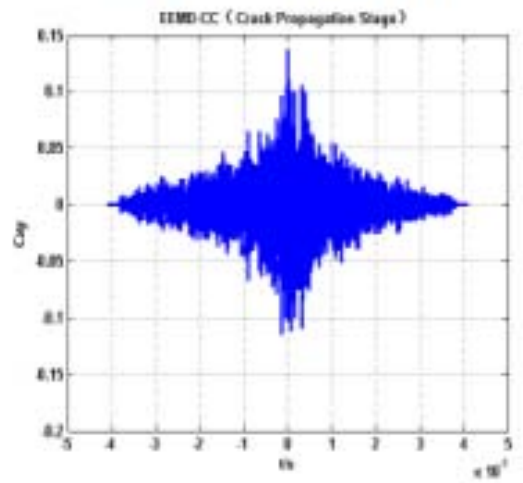

(d) EEMD-FastICA-CC $(\tau=1.8 e-006)$

Fig. 13 Time delay of four different algorithms.

Table 3 The results of time delay estimation and location calculation in different methods.

\begin{tabular}{lll}
\hline Crack signal & Time delay $(\mathrm{s})$ & $\begin{array}{l}\text { Source location } \\
(\mathrm{mm})\end{array}$ \\
\hline $\mathrm{CC}$ & $\tau=1 e-005$ & 103.5310 \\
EMD-CC & $\tau=1.8 e-005$ & 147.4185 \\
EEMD-CC & $\tau=1 e-005$ & 147.4701 \\
EEMD-FastICA-CC & $\tau=1.8 e-006$ & 149.4540 \\
\hline
\end{tabular}

\subsection{Compared to Other Algorithms}

To further verify the feasibility of the proposed algorithm, the results compared to the other algorithms such as CC, EMD-CC, EEMD-CC are as follow. Taking the crack propagation stage for example, seen from Fig. 13 and Table. 3, four different signal processing algorithms are corresponding to the four different source location results. And compared to the traditional algorithm, the proposed EEMD-FastICA $\mathrm{CC}$ algorithm spends less time delay and obtains much higher positioning accuracy.

\section{Conclusions}

In this study, focusing on the characteristics of $\mathrm{AE}$ signals Non-Stationary and time delay estimation accuracy under the uncertain correlated noises interference condition, the EEMD-Fast-ICA-CC acoustic emission source location method is proposed in this paper. At first, Fast-ICA algorithm is adopted in blind source separation of AE source signal by using the EEMD signal processing algorithm, and the main feature IMF signals are extracted as the effective acoustic emission source location signal. On this basis, time delay is obtained at different channels in combination with the cross-correlation time delay estimation principle, and the EEMD-Fast-ICA-CC source location algorithm is proposed, reducing the interference noises caused by $\mathrm{AE}$ testing and improving the positioning accuracy. Then, the effectiveness of the proposed algorithm is verified by conducting the 
three-point bending loading experiment in Q235B steel plate. Finally, compared to the traditional algorithms such as CC, EMD-CC, EEMD-CC, the location accuracy of the proposed EEMD-Fast-CA-CC algorithm obtains an obvious improvement.

\section{Acknowledgments}

This work is supported by the National Natural Science Foundation of China (51575101, 51305176), the funds of High-level talents of JIT (JIT6201623, FHXM201608).

\section{References}

[1] Rindorf, H. 1981. "Acoustic Emission Source Location in Theory and in Practice." Bruel and Kjaer Technical Review 2: 3-44.

[2] Jiang, Y., Xu, F., and Xu, B. 2015. "Acoustic Emission Tomography Based on Simultaneous Algebraic Reconstruction Technique to Visualize Damage Source Location in Q235B Steel Plate.” Mechanical System and Signal Processing 65: 452-64.

[3] Norden, H. E., and Zhaohua, W. 2009. "Ensemble Empirical Mode Decomposition: A Noise-assisted Data Analysis Method." World Scientific 1: 1-41.

[4] Zhaohua, W., and Norden, H. E. 2009. "The Multi-dimensional Ensemble Empirical Mode Decomposition Method." Advances in Adaptive Data Analysis 1: 339-72.

[5] Jian, T., and Zhao, L. J. 2011. "Vibration Analysis Based on Empirical Mode Decomposition and Partial Least Square." Procedia Engineering 16: 646-52.

[6] George, G., Loutas, T., and Stylios, C. 2013. "Bearing
Fault Detection Based on Hybrid Ensemble Detector and Empirical Mode Decomposition." Mechanical Systems and Signal Processing 16: 1-9.

[7] Zvokelj, M. 2010. "Multivariate and Multiscale Monitoring of Large-Size Low-Speed Bearings Using Ensemble Empirical Mode Decomposition Method Combined with Principal Component Analysis." Mechanical System and Signal Processing 24: 1049-67.

[8] Jin, Z. 2013. “Acoustic Emission Locatization Technique Based on Generalized Cross Correlation Time Difference Estimstion Algorithm." Chinese Journal of Sensors and Actuators 26 (11): 1513-8.

[9] Ryan, D., and James, F. K. 2005. "The Use of a Masking Signal to Improve Empirical Mode Decomposition." International Conference on Acoustics, Speech and Signal Processing 1: 485-8.

[10] Tang, B. P., and Dong, S. J. 2012. "Method for Eliminating Mode Mixing of Empirical Mode Decomposition Based on the Revised Blind Source Separation.” Signal Processing 92: 248-58.

[11] Muñoz, C. G., and Márquez, F. G. 2016. “A New Fault Location Approach for Acoustic Emission Techniques in Wind Turbines.” Energy \& Fuels 9 (1): 40.

[12] Li, X., and Adali, T. 2013. "Noncircular Complex ICA by Generalized Householder Reflections." IEEE Trans. Signal Process. 61 (24): 6423-30.

[13] Dermoune, A., and Wei, T. 2013. "FastICA Algorithm: Five Criteria for the Optimal Choice of the Nonlinearity Function." Signal Processing IEEE Transactions on 61 (8): 2078-87.

[14] Fu, X., Sidiropoulos, N., Trante, J., and Ma, W. 2015. “A Factor Analysis Framework for Power Spectra Separation and Multiple Emitter Localization." Signal Processing IEEE Transactions on 63 (24): 6581-94. 